\title{
Quality improvement and emergency laparotomy care: what have we learnt from recent major QI efforts?
}

\author{
Authors: Tim Stephens, ${ }^{A}$ Carolyn Johnston ${ }^{B}$ and Sarah Hare ${ }^{C}$
}

\begin{abstract}
More than 1.53 million adults undergo inpatient surgery in the UK NHS. Patients undergoing emergency abdominal surgery have a much greater risk of death than patients admitted for elective surgery. Widespread variations in key standards of care between hospitals exist and are associated with differences in mortality rates.

Recently there have been three large-scale initiatives to improve quality of care for emergency laparotomy patients: the National Emergency Laparotomy Audit, the enhanced perioperative care for high-risk patients trial and the Emergency Laparotomy Collaborative. Here we provide a critical review of what we currently know about the use of structured methods for improving the quality of healthcare services, with reference to the three initiatives. We find that using structured methods to improve care is the hallmark of quality improvement but attention must too be paid to the context in which these methods are used.
\end{abstract}

KEYWORDS: Postoperative care/methods, quality improvement, surgical procedures, operative mortality

\section{Introduction}

More than 1.53 million adults undergo inpatient surgery in the UK NHS each year with a 30 -day mortality of $1.5 \%{ }^{1}$. However, patients undergoing emergency abdominal surgery have a much greater risk of death. ${ }^{2,3}$ Latest figures show around 25,000 patients undergo these procedures in NHS hospitals each year, with 30-day mortality rates of $9.6 \% .{ }^{2}$ Widespread variations in key standards of care between hospitals exist, including the involvement of senior surgeons and anaesthetists and postoperative admission to critical care. These variations have been associated with differences in mortality rates. ${ }^{2-4}$

Authors: A research fellow, Barts and The London School of Medicine and Dentistry, Queen Mary University of London, London, UK; ${ }^{B}$ anaesthetist, St Georges University Hospital NHS Trust, London, UK and quality improvement lead, National Emergency Laparotomy Audit, Royal College of Anaesthetists, London, UK; Canaesthetist, Medway Maritime Hospital, Kent, UK and clinical lead, National Emergency Laparotomy Audit, Royal College of Anaesthetists, London, UK
There have been significant efforts focused on addressing these issues, both within the UK and internationally. ${ }^{2,5-8}$ In the UK, there have been three large scale initiatives to improve quality of care for emergency laparotomy patients: the National Emergency Laparotomy Audit (NELA), the enhanced perioperative care for high-risk patients (EPOCH) trial and the Emergency Laparotomy Collaborative (ELC). Although different in many aspects, at their hearts all three projects focus on using quality improvement (QI) methods to improve key care processes and, ultimately, patient outcomes. QI involves systematic efforts to reduce unwarranted variation in healthcare, using structured methods to make the changes that will lead to better system performance and better patient outcomes. ${ }^{9}$ There are good examples where discrete QI interventions have been associated with improved patient outcomes but others have yielded disappointing results, especially for complex interventions requiring coordinated change across complex hospital systems. ${ }^{10-14}$ There are now growing calls for a more critical appraisal of QI initiatives, so that the science of improvement can be more robustly developed..$^{15}$ At a national level, during the lifetime of these projects the 30-day mortality associated with emergency laparotomy has decreased from a historical figure of $14.9 \%$ to $11.8 \%$ by the end of the first year of NELA, and to $9.5 \%$ in 2018 . $^{2,3,16}$ This is an important and positive development for this patient group. However, the specific contribution of QI methodology to this reduction in mortality is challenging to ascertain due to the multiple different project designs, with overlapping time periods and differing results obtained from the two intervention studies (EPOCH and the $E L C)$. This is a common challenge in current improvement science research. $^{17,18}$

The objective of this paper is to provide a critical review of what we currently know about the use of structured methods for improving the quality of healthcare services, with reference to the three aforementioned UK initiatives focused on emergency laparotomy care.

\section{Three major initiatives to improve emergency general surgery}

NELA is an ongoing major national audit commissioned by the Healthcare Quality Improvement Partnership (HQIP) and funded by NHS England and the Welsh government. ${ }^{2,19}$ NELA is one of the first national audits to provide real-time data to inform clinicians about the process of patient care and subsequent outcomes at both the local (hospital) and national (NHS) level. Facilities to 
access data instantly, rather than waiting for annual reports, allowed a data driven QI approach to be used in both the EPOCH trial and ELC project. The EPOCH trial was a large stepped-wedge, cluster randomised trial of a national QI programme to implement a care pathway of 37 elements for patients undergoing emergency laparotomy, which was funded by the National Institute for Health Research. ${ }^{6}$ EPOCH used NELA data to study outcomes for almost 16,000 patients in $93 \mathrm{NHS}$ hospitals throughout the UK. While the QI intervention did not impact upon patient survival, EPOCH provided vital learning about how we can and cannot improve patient care using large-scale QI programmes. ${ }^{20,21} \mathrm{ELC}$ was a large QI project funded by the Health Foundation, adopting implementation science to improve patient care. ${ }^{7}$ The project was led by the Kent Surrey Sussex Academic Health Science Network (www.kssahsn.net) in 28 NHS hospitals, and used a quality improvement collaborative model to help teams to implement a laparotomy care bundle. Using statistical process control chart analysis, the ELC demonstrated an improvement in care-bundle compliance with a concurrent association with decreased 30-day mortality (to $8.3 \%$ ) during the course of the ELC project. ${ }^{6}$ However, the ELC was designed as a QI project rather than a clinical trial and the observational nature of the study and lack of control group mean a causal relationship between the intervention and improved outcomes cannot be confirmed.

\section{The main QI methods and their usage to improve emergency laparotomy care}

\section{Audit and feedback}

In an audit and feedback (A\&F) process, an individual's or team's performance is measured and then compared with agreed professional standards or targets. The results of this comparison are then fed back to the individual or team with the aim of encouraging greater adherence to the desired professional standards. ${ }^{22,23}$ Most A\&F has been focused at the individual clinician level and the most recent Cochrane systematic review of the evidence found this methodology can lead to small but potentially important improvements in professional practice. ${ }^{22}$ Effectiveness seems to depend on baseline performance, with poor baseline performance more amenable to change, and also on how the feedback is provided. Studies of team-based A\&F were absent from the 2012 Cochrane Review but there is a small but emerging body of research suggesting that a team-based approach can be effective, although the mechanisms of effect may be different from individual-level feedback. ${ }^{24-26}$

In the efforts to improve emergency laparotomy care, A\&F has been facilitated by the data presentation tools provided by the NELA team, and team-based A\&F has been promoted by all three improvement projects as a key strategy to motivate teams to improve local care processes. However, challenges exist in delivering effective $A \& F$, including difficulties in data collection and a lack of formal opportunities for feedback resulting in data feedback that is not contemporaneous; healthcare teams are generally considered pseudo-teams and so team members receiving the feedback may or may not have been those involved in the performance under review; ${ }^{27}$ and some performance feedback may be regarding key standards that are outwith of the team's ability to influence, eg access to emergency theatres limited by capacity issues. All the consequences of these challenges are likely to weaken the potential impact of A\&F on process improvement. $^{22,26}$
Model for Improvement and plan, do, study, act cycles

The Model for Improvement focuses on using data to understand current performance, setting clear, measurable goals and then developing potential solutions to achieve these goals. ${ }^{28}$ The plan, do, study, act (PDSA) cycle promotes rapid cycle testing of these potential solutions, re-evaluating performance on a regular basis and adjusting solutions iteratively based on that review. The four stages mirror the scientific experimental method of formulating a hypothesis, collecting data to test this hypothesis, analysing and interpreting the results and making inferences to iterate the hypothesis. ${ }^{29}$ Unlike A\&F, which has been extensively researched, there has been little empirical evaluation of this approach. The research that does exist, on the PDSA cycle specifically, suggests clinical staff often find PDSAs difficult to carry out in the methodical fashion intended, often with consequently disappointing results. ${ }^{29,30}$

Use of NELA data can facilitate situational awareness and support tracking of progress with improvement solutions (the 'How will we know a change is an improvement?' question in the Model for Improvement). In all three projects, the clinical standards or target care processes were prescribed eg patients should enter the operating theatre within their target National Confidential Enquiry into Patient Outcome and Death time-frame or patients should be admitted to a critical care unit postoperatively. The challenge for those clinicians leading change locally was therefore not 'what to improve' in order to reduce mortality for these patients, but 'how to improve' care locally in line with these standards. Time constraints inherent in clinician-led QI, plus, in the case of the $\mathrm{EPOCH}$ trial, the complexity of the care pathway meant that the experience of the EPOCH/ELC teams mirrored that of the extant research. Frontline teams did not receive sufficient training to use the approach and/or did not have sufficient time in their working week to methodically develop, test and refine potential solutions that would lead to improved care. This was a major theme of the evaluation of the EPOCH trial in particular (explored further below). ${ }^{20}$ Another recent controlled evaluation in another aspect of emergency surgery (the CholeQuIC project) found that the time spent on deliberation upfront made for better solutions that needed fewer rounds of testing, but that an openness within the team to testing and iterative adaptation was also vital. ${ }^{31}$ Thus acting 'scientifically and pragmatically' through a trial-and-errorbased approach may be more palatable to busy frontline clinicians than the PDSA cycle approach. ${ }^{32}$

\section{QI collaborative approach}

A QI collaborative (QIC) is an organised, multifaceted approach that includes teams from multiple healthcare sites coming together to learn, apply and share improvement methods, ideas and data on service performance for a given healthcare topic. $^{33}$ Although sites may use methods such as A\&F or the Model for Improvement to effect change locally, the added value of the collaborative approach is thought to be the creation of a new cooperative space for clinicians to share and learn together outside their normal working environment. ${ }^{34} \mathrm{~A}$ recent systematic review found that 53 of the 64 studies ( $83 \%$ ) that met the Effective Practice and Organisation of Care study design standards for inclusion found measured improvements in at least one target process. Collaboratives reporting success generally 
addressed relatively straightforward aspects of care, had a strong evidence base and noted a clear evidence-practice gap in an accepted clinical pathway or guideline. ${ }^{34}$ Notably, only one of the collaboratives focused on perioperative care. ${ }^{35}$

The ELC was based on a QIC approach with multiple collaborative meetings, both cohort-wide and regional meetings within the cohort, and webinars/calls over the 2-year project period. Conversely, the EPOCH trial followed a more 'light touch' QI campaign approach, with the focus on awareness-raising and basic QI skills training. Face-to-face contact was relatively limited, with additional support and resources available online. The QI campaign is a successful approach to QI, but it is likely, based on the results of the EPOCH trial, that it is not suited to complex quality problems, even in the presence of accepted evidencebased guidelines as the hard core of the intervention. ${ }^{20,36}$

Other QI approaches, such as Lean and Six Sigma are used in the NHS but were not used in any of the three emergency surgery projects so are not included in this review. ${ }^{37}$

\section{Beyond QI methods: the influence of context on QI in emergency surgery}

Context characterises the overall environment in which QI activities take place and includes prevailing national policies, local organisational structure, the culture of an organisation overall and of the specific departments within an organisation. ${ }^{38}$ Contextual factors are distinct from the clinical and QI interventions within an improvement project but are highly influential on the success or otherwise of such projects. ${ }^{39}$

Recent work by the NELA group has identified that a substantial amount of the observed variation in mortality nationally was explained by differences in hospital structures and characteristics, such as the number of operating theatres available or the existence of an emergency surgical unit. ${ }^{4}$ The work of Donabedian on the relationship between structure, process and outcome remains highly relevant and QI methods are best suited to tackling process, rather than structural quality problems. ${ }^{40}$ For example, improving the process by which emergency patients flow through theatres could be amenable to QI methods, but increasing the number of theatres available (with commensurate staffing and equipment) is an organisational management issue, requiring additional funding, recruitment and procurement. It is perhaps notable that across all three initiatives, improving the time for getting emergency laparotomy patients into theatre has remained the most stubbornly challenging aspect of care to improve, suggesting that it may not amenable to improvement by QI methods alone. 2,6,7

Consideration of context goes beyond the structural aspects of an organisation. In the EPOCH trial, limited resources, both human and financial, and organisational upheaval were cited as key challenges by many interviewed in the process evaluation, as was lack of engagement of colleagues and hospital executives. ${ }^{20}$ One significant consequence of this may have been a lack of organisational support for NELA data collection in around half of the EPOCH trial sites. A non-supportive context meant that the burden of data collection fell to a few clinicians leading the local improvement projects. While data is central to any QI project, it is the use of this data through feedback, combined with other improvement strategies, that is likely to achieve more robust results. ${ }^{22,41-43}$ If future QI programmes are to capitalise on concurrent national audits or other ongoing data collection, contextual factors need to be addressed to allow embedding of data collection processes well before the start of any improvement work. This may take considerably longer than anticipated. ${ }^{41}$

\section{Interpretation and discussion}

This review, particularly in the context of these three initiatives, demonstrates the challenge of QI in healthcare. Using structured approaches to improvement is the hallmark of QI but attention must too be paid to the context - at the system/policy, hospital and team levels. Due to the time and effort required to effectively apply such methods, and the need for colleagues to change behaviours or practice as a result, an unsupportive context may stymie even the most rigorously applied QI methods. The impact of time and resources also needs to be recognised. Resource is required not only at the national or regional improvement project level, to focus attention and support change, but at the individual hospital level to unblock contextual barriers to improvement. While ostensibly the ELC did not provide hospitals with any more resources than the EPOCH trial QI programme, the impact of NELA (and indeed the EPOCH trial itself) was likely to have generated a more receptive context for improvement in emergency general surgery, with a greater awareness of the problem, not least among senior and middle management in hospitals, and regional and national policymakers. The ELC, with a 2-year project period, also provided more time for change to occur than the EPOCH trial. To that end, improvement-focused national audits such as NELA may prove to be the most effective centrally organised approach to the improvement of complex quality issues due to the open ended time period (rather than a time-constrained 'project'), and also due to the potential impact on structural issues in the medium to longer term, such as the number of operating theatres, through national reporting and benchmarking. However, data needs to be used locally to effect change and that means time to focus on improvement must be included in job plans for clinical staff and frontline managers, allied with appropriate training in QI skills. QI is not a quick and easy fix for complex quality problems. As such, using QI to improve emergency surgical care, indeed any complex system issue in healthcare, requires not only the right QI 'method' but also time, commitment and resource from healthcare organisations if efforts are to lead to actual improvements in patient outcomes.

\section{Conflicts of interests}

Carolyn Johnston and Sarah Hare both receive salary support for their roles within the National Emergency Laparotomy Audit. Tim Stephens received salary support for his roles in the EPOCH trial and Emergency Laparotomy Collaborative.

\section{References}

1 Abbott TEF, Fowler AJ, Dobbs TD et al. Frequency of surgical treatment and related hospital procedures in the UK: a national ecological study using hospital episode statistics. Br J Anaesth 2017:119:249-57.

2 The National Emergency Laparotomy Project Team. Fourth patient report of the National Emergency Laparotomy Audit. London: Royal College of Anaesthetists, 2018.

3 Saunders DI, Murray D, Pichel AC, Varley S, Peden CJ. Variations in mortality after emergency laparotomy: the first report of the UK Emergency Laparotomy Network. Br ] Anaesth 2012;109:368-75.

4 Oliver CM, Bassett MG, Poulton TE et al. Organisational factors and mortality after an emergency laparotomy: multilevel analysis of 39903 National Emergency Laparotomy Audit patients. Br J Anaesth 2018;121:1346-56. 
5 Huddart S, Peden C], Swart M et al. Use of a pathway quality improvement care bundle to reduce mortality after emergency laparotomy. Br J Surg 2015;102:57-66.

6 Peden C], Stephens T], Martin G et al. Effectiveness of a nationa quality improvement programme to improve survival after emergency abdominal surgery (EPOCH): A stepped-wedge cluster randomised trial. Lancet 2019;393:2213-21.

7 Aggarwal G, Peden C], Mohammed MA et al. Evaluation of the Collaborative use of an evidence-based care bundle in emergency laparotomy. JAMA Surg 2019;154:1-9.

8 Vester-Andersen M, Lundstrom LH, Moller MH et al. Mortality and postoperative care pathways after emergency gastrointestinal surgery in 2904 patients: A population-based cohort study. $\mathrm{Br}$ ] Anaesth 2014:112:860-70.

9 Batalden PB, Davidoff F. What is 'quality improvement' and how can it transform healthcare? Qual Saf Heal Care 2007:16:2-3.

10 Haynes AB, Weiser TG, Berry WR et al. A surgical safety checklist to reduce morbidity and mortality in a global population. New Engl J Med 2009:360:491-9.

11 Pronovost PJ, Watson SR, Goeschel CA, Hyzy RC, Berenholtz SM. Sustaining reductions in central line-associated bloodstream infections in Michigan intensive care units: a 10-year analysis. Am J Med Qual 2016;31:197-202.

12 Benning A, Dixon-Woods M, Nwulu U et al. Multiple component patient safety intervention in English hospitals: controlled evalua tion of second phase. BMJ 2011;342:d199.

13 Power M, Tyrrell PJ, Rudd AG et al. Did a quality improvement collaborative make stroke care better? A cluster randomized trial. Implement Sci 2014;9:40.

14 Bion J, Richardson A, Hibbert P et al. 'Matching Michigan': a 2-year stepped interventional programme to minimise central venous catheter-blood stream infections in intensive care units in England. BMJ Qual Saf 2013;22:110-23.

15 Dixon-Woods M, Martin GP. Does quality improvement improve quality? FHJ 2016:3:191-4.

16 The National Emergency Laparotomy Project Team. First patient report of the National Emergency Laparotomy Audit. London: Royal College of Anaesthetists, 2015.

17 Grady D, Redberg RF, O'Malley PG. Quality improvement for quality improvement studies. JAMA Intern Med 2018;178:187.

18 Poots AJ, Reed JE, Woodcock T, Bell D, Goldmann D. How to attribute causality in quality improvement : lessons from epidemiology. BMJ Qual Saf 2017;26:933-7.

19 The National Emergency Laparotomy Project Team. Second patient report of the National Emergency Laparotomy Audit. London: Royal College of Anaesthetists, 2016.

20 Stephens TJ, Peden C], Pearse RM et al. Improving care at scale: process evaluation of a multi-component quality improvement intervention to reduce mortality after emergency abdominal surgery (EPOCH trial). Implement Sci 2018;13:142.

21 Martin GP, Kocman D, Stephens T, Peden C], Pearse RM. Pathways to professionalism? Quality improvement, care pathways, and the interplay of standardisation and clinical autonomy. Sociol Heal IIln 2017;39:1314-29.

22 Ivers N, Jamtvedt G, Flottorp S et al. Audit and feedback : effects on professional practice and healthcare outcomes. Cochrane Database Syst Rev 2012:CD000259.

23 Hysong SJ, Best RG, Pugh JA. Audit and feedback and clinical practice guideline adherence: making feedback actionable. Implement Sci 2006;1:9.

24 Hysong SJ, Knox MK, Haidet P. Examining clinical performance feedback in patient-aligned care teams. J Gen Intern Med 2014;29(Suppl 2):S667-74.

25 Hysong S], Francis ], Petersen LA. Motivating and engaging frontline providers in measuring and improving team clinical performance. BMJ Qual Saf 2019;28:405-11.
26 Brown B, Gude WT, Blakeman T et al. Clinical Performance Feedback Intervention Theory (CP-FIT): A new theory for designing, implementing, and evaluating feedback in health care based on a systematic review and meta-synthesis of qualitative research. Implement Sci 2019;14:1-25.

27 West MA, Lyubovnikova J. Illusions of team working in health care. J Health Organ Manag 2013;27:134-42.

28 Institute for Healthcare Improvement. The Breakthrough Series: IHI's Collaborative Model for Achieving Breakthrough Improvement. Boston: IHI, 2003.

29 Taylor M], McNicholas C, Nicolay C et al. Systematic review of the application of the plan-do-study-act method to improve quality in healthcare. BMJ Qual Saf 2014;23:290-8.

30 Mcnicholas C, Lennox L, Woodcock T, Bell D, Reed JE. Evolving quality improvement support strategies to improve plan-do-studyact cycle fidelity: A retrospective mixed-methods study. BMJ Qual Saf 2019:28:356-65.

31 Stephens T], Bamber JR, Beckingham IJ et al. Understanding the influences on successful quality improvement in emergency general surgery: learning from the RCS CholeQuIC project. Implement Sci 2019;4:84

32 Reed JE, Howe C, Doyle C, Bell D. Simple rules for evidence translation in complex systems: A qualitative study. BMC Med 2018;16:92.

33 Schouten LMT, Hulscher MEJL, van Everdingen JJE, Huijsman R, Grol RPTM. Evidence for the impact of quality improvement collaboratives: systematic review. BMJ 2008;336:1491-4.

34 Wells S, Tamir O, Gray ] et al. Are quality improvement collaboratives effective? A systematic review. BMJ Qual Saf 2018;27:22640.

35 Campbell DA. Accelerating the pace of surgical quality improvement. Arch Surg 2010:145:985

36 Khodyakov D, Ridgely MS, Huang C, DeBartolo KO, Sorbero ME, Schneider EC. Project JOINTS: What factors affect bundle adoption in a voluntary quality improvement campaign? BMJ Qual Saf 2015;24:38-47.

37 McCulloch P, Kreckler S, New S et al. Effect of a 'Lean' intervention to improve safety processes and outcomes on a surgical emergency unit. BMJ 2010;341:c5469.

38 Kaplan HC, Provost LP, Froehle CM, Margolis PA. The Model for Understanding Success in Quality (MUSIQ): building a theory of context in healthcare quality improvement. BMJ Qual Saf 2012;21:13-20

39 Ogrinc G, Davies L, Goodman D et al. SQUIRE 2.0 (Standards for QUality Improvement Reporting Excellence): revised publication guidelines from a detailed consensus process. BMJ Qual Saf 2015;25:986-92.

40 Donabedian A. The quality of care: How can it be assessed? J Med Assoc Thail 1988;260:1743-8.

41 Dixon-Woods M, McNicol S, Martin G. Ten challenges in improving quality in healthcare: lessons from the Health Foundation's programme evaluations and relevant literature. BMJ Qual Saf 2012;21:876-84.

42 Dixon-Woods M, Bosk CL, Aveling EL, Goeschel C, Pronovost PJ. Explaining Michigan: developing an ex post theory of a quality improvement program. Milbank Q 2011;89:167-205.

43 Greenhalgh T, Robert G, Macfarlane F, Bate P, Kyriakidou 0. Diffusion of innovations in service organizations: systematic review and recommendations. Milbank Q 2004;82:581-629.

Address for correspondence: Tim Stephens, Critical Care and Perioperative Medicine Research Group, William Harvey Research Institute, Queen Mary University of London, c/o ACCU Research Team, Royal London Hospital, London E1 1BB, UK. Email: t.t.stephens@qmul.ac.uk 